\title{
Robotic System for No-Scar Gastrointestinal Surgery
}

\author{
L. Phee, Member, IEEE, S.C. Low, Z.M. Thant, K.Y. Ho and S.C. Chung
}

\begin{abstract}
In line with minimally invasive surgery (MIS), flexible endoscopy is used to inspect and treat disorders of the gastrointestinal (GI) tract without the need for creating an artificial opening on the patient's body. Simple surgical procedures (like polypectomy and biopsy) can be performed by introducing a flexible tool via a working channel to reach the site of interest at the distal end. More technically demanding surgical procedures like hemostasis for arterial bleeding, and suturing to mend a perforation cannot be effectively achieved with conventional flexible endoscopy. The proposed masterslave robotic system enables the endoscopist to perform technically demanding therapeutic procedures which are currently performed only by surgeons in an opened surgery setting. The robotic system consists of a master console and a slave. The latter is a cable driven flexible robotic manipulator that can be inserted into the tool channel of existing endoscopes or attached in tandem to the endosopes. Together with the real time endoscopic view, the endoscopist would be capable of performing more intricate and difficult surgical procedures using natural orifices to access the gastrointestinal tract thus eliminating any scars on the patient.
\end{abstract}

Keywords: surgery, robotics, master-slave, endoscope

\section{INTRODUCTION}

$I^{\prime}$ $\mathrm{n}$ line with minimally invasive surgery (MIS), flexible endoscopy is used to inspect and treat disorders of the gastrointestinal (GI) tract without the need for creating an artificial opening on the patient's body [1]. The endoscope is introduced via the mouth or anus into the upper or lower GI tracts respectively. A miniature camera at the distal end captures images of the GI wall that help the clinician in his/her diagnosis of the GI diseases. Simple surgical procedures (like polypectomy and biopsy) can be performed by introducing a flexible tool via a working channel to reach the site of interest at the distal end. The types of procedures that can be performed in this manner are limited by the lack of maneuverability of the tool. More technically demanding surgical procedures like hemostasis for arterial bleeding, suturing to mend a perforation, fundoplication for gastrooesophageal reflux cannot be effectively achieved with flexible endoscopy. These procedures are often presently being performed under opened or laparoscopic surgeries.

Manuscript received June 30, 2006. This work was supported in part by the National Medical Research Council (Singapore) under Grant NMRC/0827/2004 and Nanyang Technological University (Singapore) under grant SUG 44/04.

L. Phee, S.C. Low and Z.M. Thant are with the BioRobotics Lab, School of Mechanical \& Aerospace Engineering, Nanyang Technological University, Singapore. (phone: 65-6790 4959; fax: +65-6791 1859; e-mail: msjphee@ntu.edu.sg ).

K.Y. Ho and S.C. Chung are with the Yong Loo Lin School of Medicine, National University of Singapore.
With the invention of medical robots like the Da Vinci [2] surgical systems, clinicians are now able to maneuver surgical tools accurately and easily within the human body [3]. Operating from a master console, the clinician is able to control the movements of laparoscopic surgical tools real time. These tools (also known as the slaves) are designed with sufficient degrees of freedom to move according to the natural hand and wrist motion allowing the clinician to perform intricate procedures with minimal technical difficulties. They are also likely to be small so that they could be easily threaded through small openings or natural orifices in the patient.

The master device is the human-machine interface of the robotic system. Normally directly operated by the clinician, the master device would record and analyze the movements of the clinician's arms and hands. The processed information would be used to drive the slave manipulators. The two basic requirements for the master device are ergonomics and haptics. Large and singularity free workspace, low inertia, small backlash and force reflection are also main characteristics for a good master device [4], [5]. The master controllers for commercialized Da Vinci and ZEUS systems are ergonomics-based devices with gross force feedback [6]. Haptics-based master devices for MIS system are under intensive research [7], [8]. The designs of master device for teleoperation can also be categorized into exoskeleton and non-exoskeleton types. Most of the master devices (Da Vinci, ZEUS and PHANTOM) are nonexoskeleton structures. They enjoy little restriction on design and easier haptics incorporation. TELESAR II [9] and F-EAM [10] master devices are wearable exoskeleton structures. They enjoy better user interaction and perception over control of anthropomorphic manipulators.

This paper presents a master-slave robotic system for GI surgery. The main novelty of the system is the flexible transmission structure to the slave manipulators. This feature would enable the slave to be introduced in tandem with a flexible endoscope via natural orifices (e.g. mouth and anus) to excess the GI tract to perform dexterous procedures.

\section{II.OVERVIEW OF ROBOTIC SYSTEM}

Figure 1 shows the proposed system layout whereby the endoscopist works on the master console while gathering visual feedback from the endoscope. A computer console will interpret the readings from the master console that will in turn give instructions to the slave robotic system to perform the treatment to the patient. This system allows 


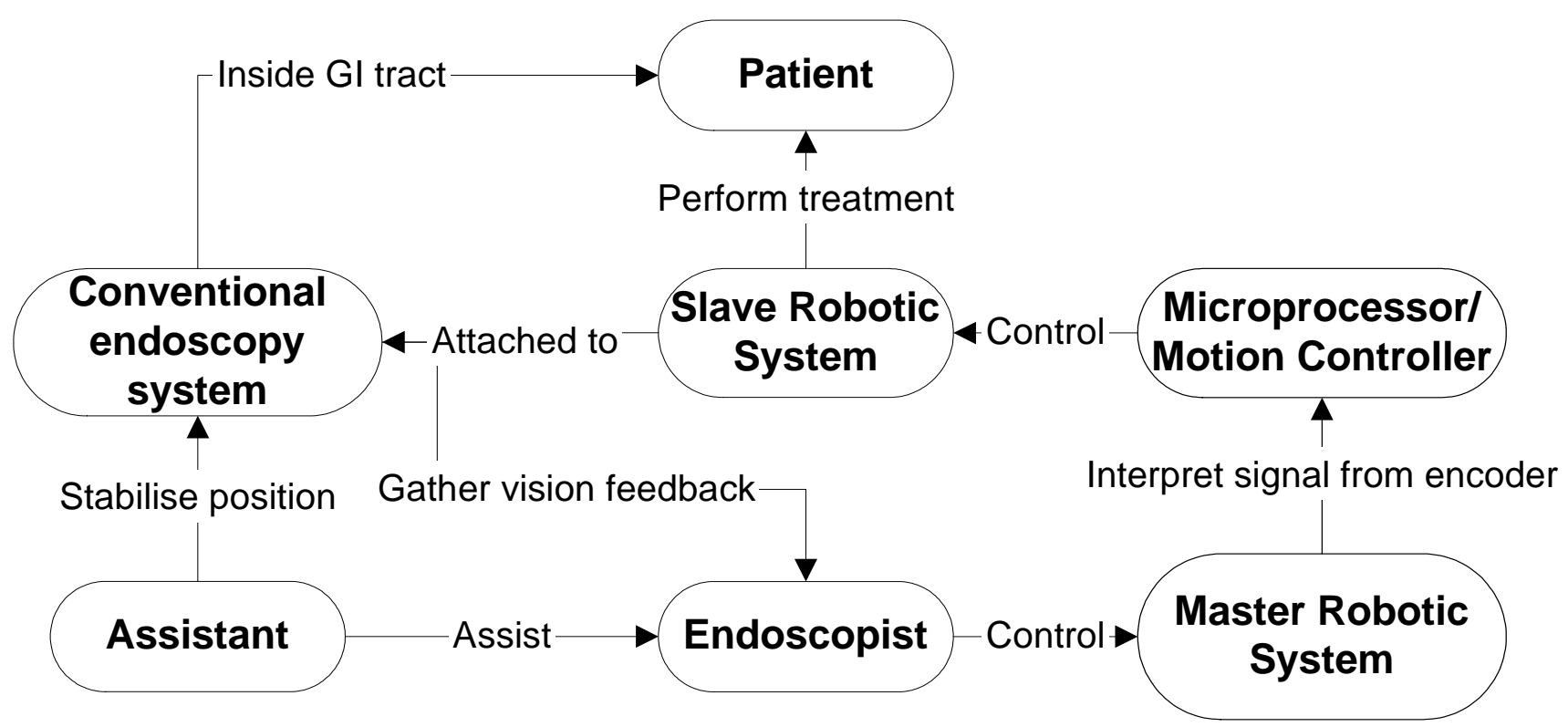

Fig. 1. Proposed System Layout

complicated treatment to be performed with the added benefit of easy and intuitive control for the endoscopist.

\section{A. The Slave Manipulator}

The 3D model of the intended slave manipulator can be seen in Fig. 2.

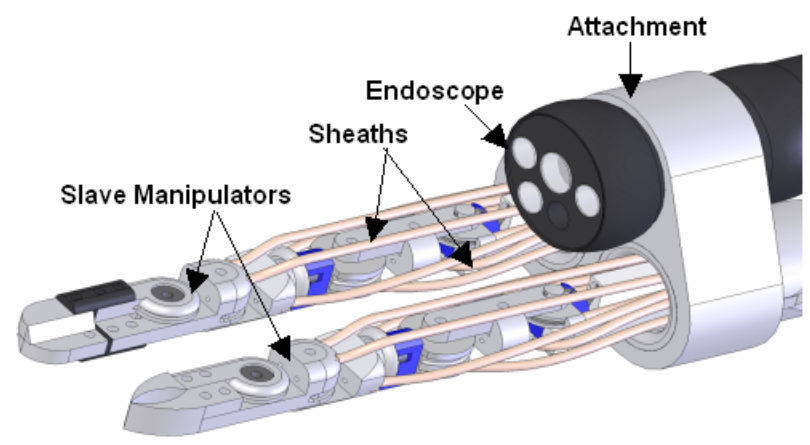

Fig. 2. 3D model of slave manipulators attached with the endoscope.

In order for the surgeon to perform the necessary dexterous actions, the slave manipulator possesses a high number of Degrees of Freedom (DOFs). The emphasis of the project is to make the slave manipulator to be as intuitive to control as possible. As such, the DOFs and joints of the slave manipulator are modeled after a simplified human arm as shown in Fig 3. Altogether there are 5 DOFs for positioning of the slave and an extra DOF for manipulating the end effector and the axis or rotation. Two slave manipulators are used instead of one since it can perform actions such as pulling and cutting of polyps or suturing bleeding sites. Furthermore two slave manipulators are more intuitive to use since they resemble the two human arms.
In order for the slave manipulator to be able to be threaded non-invasively into the human GI tract, the overall size of the slave manipulator has to be small yet flexible. Due to this unique requirement, tendon-sheath actuation mechanism is used. The prime movers are located outside the human body and it transmits power to the mechanism by pulling and releasing flexible tendons in a flexible sheath accordingly.

The design of the mechanism is such that the two antagonistic tendons that control each DOF motion are independently actuated by one DC motor. In addition, the rotational displacement of each joint is directly proportional to the linear displacements of the tendon. These characteristics allow the serial slave manipulator to be controlled more easily.

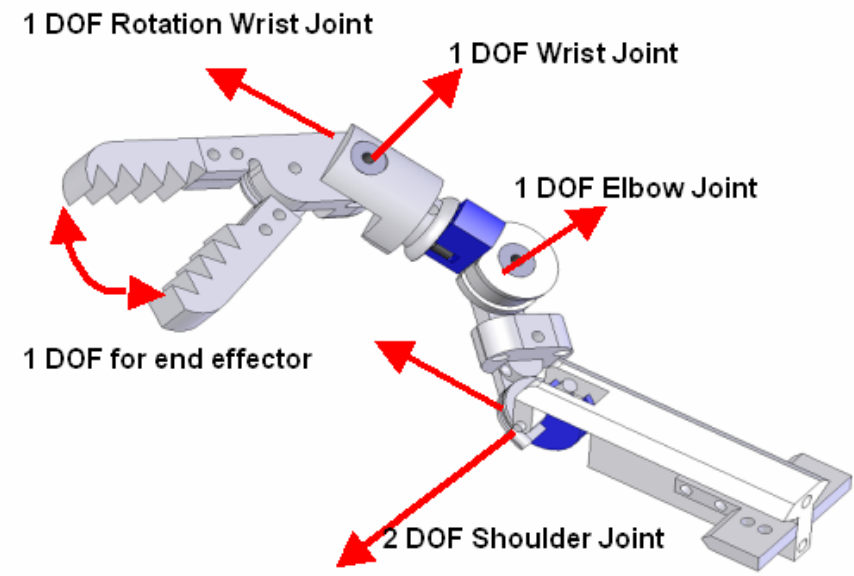

Fig. 3. The degrees of freedoms for one arm of the slave manipulator 
Different types of end effectors have been designed to perform different types of actions during surgery. One of the tools includes electrodes that can perform mono-polar cauterizing. The picture of the current developed prototype is shown in Fig. 4.

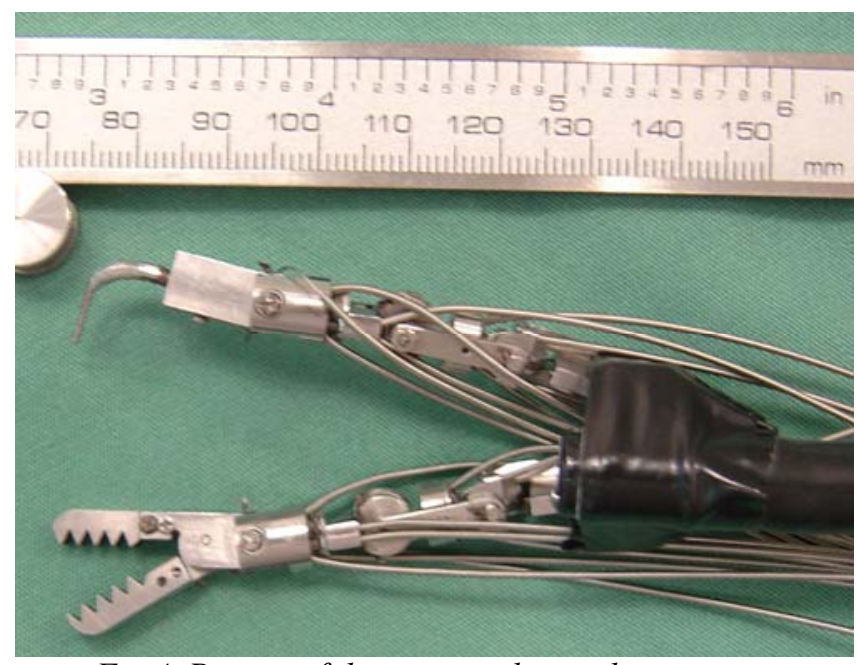

Fig 4. Picture of the current slave robotic system

\section{B. The Master Controller System}

Ergonomics plays an important part in designing the master device. There are intrinsic limitations due to the operator's capability; workspace and resolution. The master must be able to generate movements above the level of the operator's detection and provide significant information from the slave to the operator. The master should also possess higher position resolution than the slave. Its friction, mass and inertia should be low enough to give comfort to the operator.

For the exoskeleton master device, human factors must be taken into careful consideration in order to have better ergonomics. The master device has to extract all the necessary information from the operator without imposing any limitation to the operator's desired movement unless it is out of range for the slave manipulator. Anthropomorphic data and range of motion of the human arm was used in the design of the master device. The design of the exoskeleton arm master is more than quantitative problem solving. The qualitative data, such as comfort, aesthetics, user friendliness and safety, are also to be accessed during the development process. The surgeon should be seated comfortably at a master console viewing an image of the surgical field. His hands and wrists should be naturally positioned relative to his eyes. Upper arm should be positioned within $20^{\circ}$ extension and $20^{\circ}$ flexion, Lower $\operatorname{arm}$ within $60^{\circ}$ to $100^{\circ}$ flexion, and wrist within $0^{\circ}$ to $15^{\circ}$ flexion/extension [11].

As the master is to be used in surgical theatre, size, material and sensors/actuators must be selected according to the theatre conditions and requirements. Magnetic trackers/sensors cannot be used to measure the arm position due to the varying magnetic fields from different equipments in the operation theatre. The ergonomic master device was developed as a 6 DOF structure to control the 6 DOF slave mechanism. As the slave manipulators resemble the human arms, the anthropomorphic data of the surgeon's arm is used as the control parameters in order to give the surgeon better perception in performing the joint-to-joint control of the slave.

The schematic of the master controller is shown in Fig. 5. Two cable-actuated position sensors are used to measure the two contributing rotations of the shoulder joint that cause the up-down and in-out movement of the upper arm. Two optical rotary encoders are used to measure the flexion and supination-pronation movement of the elbow joint. Another similar encoder is used for flexion-hyperextension movement of the wrist, and the finger grip comes with an encoder inside the holder. A virtual plane is formed by shoulder joint and the two position sensors. The sensor locations can be modified to meet the required workspace that lies on one side of the plane. For the full range upper arm movement, additional sensor will be needed. As the comfortable workspace for the surgeon's upper arm lies in a quarter of a spherical space, two position sensors is sufficient to get the anthropomorphic data of one arm in that range.

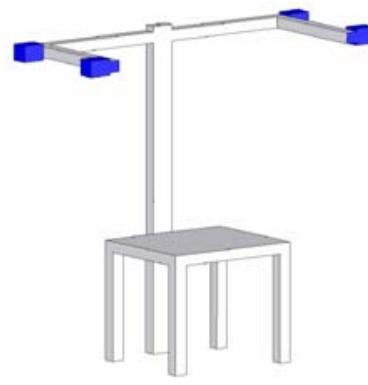

(a)

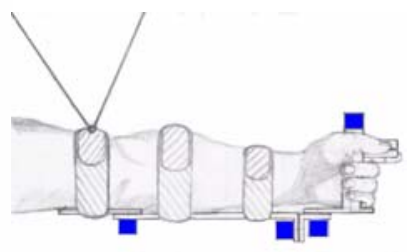

(b)
Fig. 5. The whole schematic of the master setup (a) frame with two cable sensors for each arm (b) left arm with sensors attached

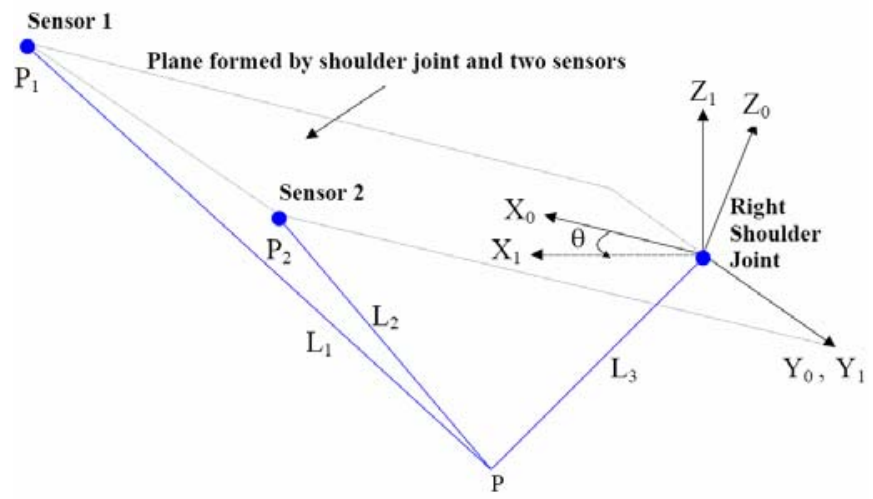

Fig. 6. The location of cable-attached upper arm in two coordinate systems 
The position sensors are located in such a way that the cables do not impose any disturbance on the surgeon's vision and movement. Furthermore the tensions of the cables facilitate the surgeon as a counterweight from his own arm moments, reducing the fatigue. Assuming that the shoulder joint is a ball and socket type and its centre estimated about the center of the ball [12], the point of cable-attached upper arm is determined by three spheres formed by upper arm length and two cable lengths. In Fig. 6, $\mathrm{L}_{1}$ and $\mathrm{L}_{2}$ are the two cable lengths and $\mathrm{L}_{3}$ is the upper arm length.

In $\mathrm{X}_{0} \mathrm{Y}_{0} \mathrm{Z}_{0}$ coordinate system,

$$
\begin{aligned}
\left({ }^{0} P_{x}-{ }^{0} P_{1 x}\right)^{2}+\left({ }^{0} P_{y}-{ }^{0} P_{1 y}\right)^{2}+\left({ }^{0} P_{z}\right)^{2} & =L_{1}^{2} \\
\left({ }^{0} P_{x}-{ }^{0} P_{2 x}\right)^{2}+\left({ }^{0} P_{y}-{ }^{0} P_{2 y}\right)^{2}+\left({ }^{0} P_{z}\right)^{2} & =L_{2}^{2} \\
\left({ }^{0} P_{x}\right)^{2}+\left({ }^{0} P_{y}\right)^{2}+\left({ }^{0} P_{z}\right)^{2} & =L_{3}^{2}
\end{aligned}
$$

The point ${ }^{0} \mathrm{P}(\mathrm{x}, \mathrm{y}, \mathrm{z})$ below the plane can be solved from the above equations. And it can be transformed into $\mathrm{X}_{1} \mathrm{Y}_{1} \mathrm{Z}_{1}$ by rotation matrix about $\mathrm{Y}$-axis.

$$
\left\{\begin{array}{c}
{ }^{1} P_{x} \\
{ }^{1} P_{y} \\
{ }^{1} P_{z}
\end{array}\right\}=\left[\begin{array}{ccc}
\cos \theta & 0 & \sin \theta \\
0 & 1 & 0 \\
-\sin \theta & 0 & \cos \theta
\end{array}\right]\left\{\begin{array}{c}
{ }^{0} P_{x} \\
{ }^{0} P_{y} \\
{ }^{0} P_{z}
\end{array}\right\}
$$

${ }^{1} \mathrm{P}(\mathrm{x}, \mathrm{y}, \mathrm{z})$ can be used to calculate the shoulder joint up-down and in-out angles.

$$
\begin{aligned}
& \theta_{\text {in-out }}=\cos ^{-1}\left(\frac{{ }^{1} P_{x}}{{ }^{1} P_{(x, y, z)}}\right) \\
& \theta_{\text {up-down }}=\cos ^{-1}\left(\frac{{ }^{1} P_{z}}{{ }^{1} P_{(x, y, z)}}\right)
\end{aligned}
$$

Table 1. Range of motion of the master

\begin{tabular}{|l|l|l|}
\hline $\begin{array}{l}\text { No } \\
:\end{array}$ & Motion & Range \\
\hline 1 & Shoulder in-out & $\theta_{\text {in-out }}$ \\
\hline 2 & Shoulder up-down & $\theta_{\text {up-down }}$ \\
\hline 3 & Elbow flexion & $0^{\circ}$ to $140^{\circ}$ \\
\hline 4 & Elbow supination/pronation & $-80^{\circ}$ to $80^{\circ}$ \\
\hline 5 & Wrist flexion/extension & $-60^{\circ}$ to $60^{\circ}$ \\
\hline 6 & Gripper & $0^{\circ}$ to $60^{\circ}$ \\
\hline
\end{tabular}

The mobility range of the master is constrained only at the upper arm by the virtual plane formed by shoulder joint and two cable sensors. Other joints satisfy full mobility range of the surgeon in their respective movements as shown in Table 1 .

The master-slave teleoperation requires kinematics transformations which relate cartesian and joint variables within master and slave devices. This section presents the DH parameters and forward kinematics transformation for the master device. The kinematics diagram and $\mathrm{DH}$ parameters [13] are shown in Fig. 7 and Table 2, respectively. $\theta(1-6)$ are the angles of rotation and $\mathrm{a} 3, \mathrm{~d} 5$ and a6 are the upper arm, lower arm and hand lengths, respectively.

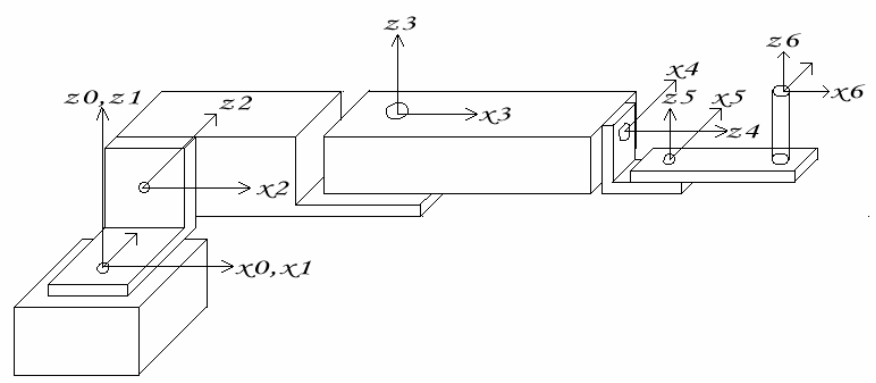

Fig. 7. Master kinematic diagram

Table 2. Master DH parameters

\begin{tabular}{|c|c|c|c|c|}
\hline $\mathrm{I}$ & $\theta(\mathrm{i})$ & $\alpha(\mathrm{i}-1)$ & $\mathrm{a}(\mathrm{i}-1)$ & $\mathrm{d}(\mathrm{i})$ \\
\hline 1 & $\theta 1$ & 0 & 0 & 0 \\
\hline 2 & $\theta 2$ & -90 & 0 & 0 \\
\hline 3 & $\theta 3$ & 90 & $\mathrm{a} 2$ & 0 \\
\hline 4 & $\theta 4+90$ & 90 & 0 & 0 \\
\hline 5 & $\theta 5$ & -90 & 0 & $\mathrm{~d} 5$ \\
\hline 6 & $\theta 6-90$ & 0 & $\mathrm{a} 5$ & 0 \\
\hline
\end{tabular}

From each row in a $\mathrm{DH}$ parameter table, the homogeneous transformation matrix is

$$
{ }_{i}^{i-1} T=\left[\begin{array}{cccc}
c \theta_{i} & -s \theta_{i} & 0 & a_{i-1} \\
s \theta_{i} c \alpha_{i-1} & c \theta_{i} s \alpha_{i-1} & -s \alpha_{i-1} & -d_{i} s \alpha_{i-1} \\
s \theta_{i} s \alpha_{i-1} & c \theta_{i} c \alpha_{i-1} & c \alpha_{i-1} & d_{i} c \alpha_{i-1} \\
0 & 0 & 0 & 1
\end{array}\right]
$$

where $c \theta_{\mathrm{i}}=\cos \left(\theta_{\mathrm{i}}\right), s \theta_{\mathrm{i}}=\sin \left(\theta_{\mathrm{i}}\right)$, ect. The forward kinematic transformation for active joints is

$$
{ }_{n}^{0} T=\prod_{i=1}^{n}{ }_{i}^{i-1} T={ }_{1}^{0} T{ }_{2}^{1} T \ldots{ }_{n}^{n-1} T
$$




\section{Software Control}

A dedicated computer software takes real time readings from each encoder of the master device. This data is processed and appropriate signals are sent out to actuate the respective motors to bring about the required movements. The system framework adopts a 2-layer architecture. The bottom layer is the hardware control module that uses lowlevel drivers for hardware control. The top-layer modules are the kernel algorithms, which perform the signal processing to compensate for backlash and noise disturbances.

\section{PRELIMINARY EXPERIMENTS}

In vitro experiments with the master-slave system have been conducted in carrying out simple manipulative tasks. By manipulating the master, users are able to command the slave to carry out pick and place actions as shown in Fig. 8. Trials with explanted organs and animal trials are being planned in the near future.

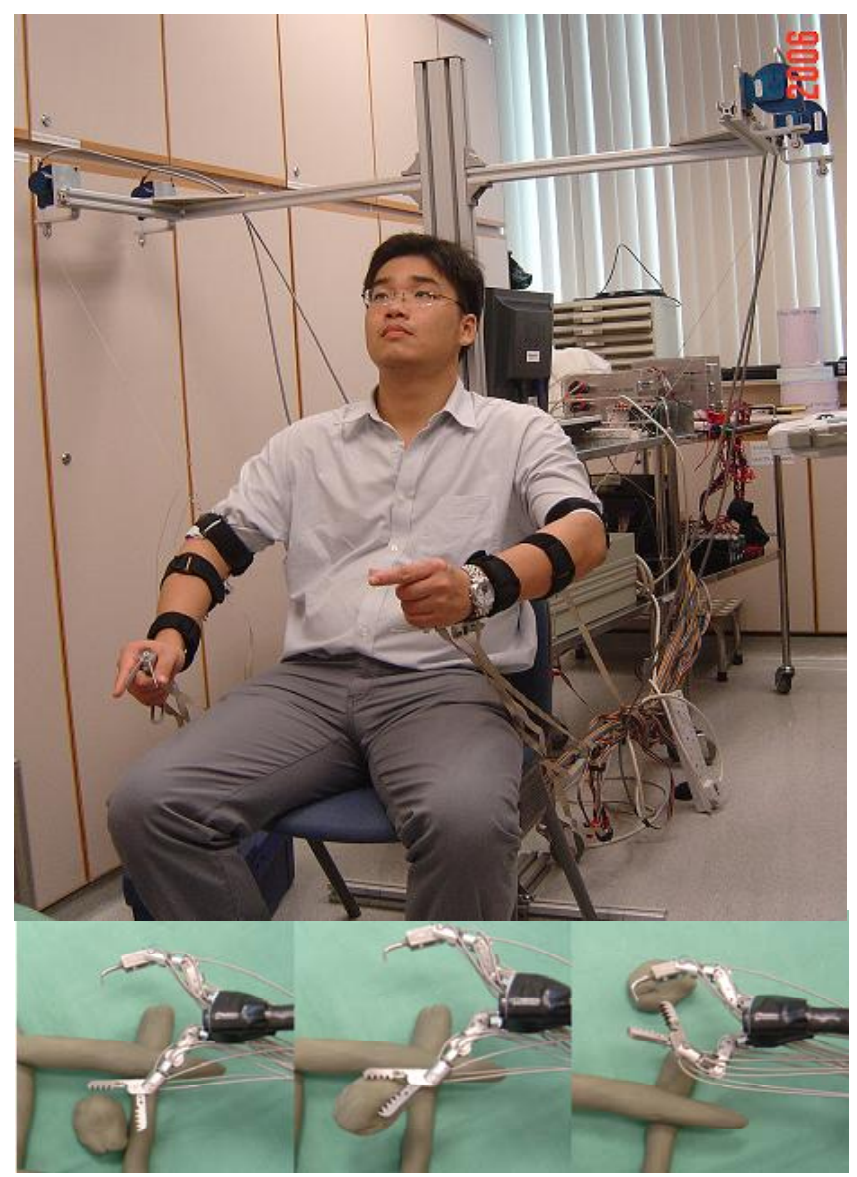

Fig. 8. Using the robotic system for pick and place tasks

\section{DISCUSSION}

One of the biggest problems in the tendon-sheath driving robot system control is the non-linear characteristics due to the friction between the tendon and sheath [5]. As a result, delays and movement hysteresis are noticeable and can be seen in the below relationship

$\delta(L)=\frac{T_{0} L}{E A} \frac{\exp (\lambda)-\lambda-1}{\lambda}$

where $T_{0}$ is tendon-pretension, $L$ is the length of the tendon-sheath, $E$ is Young's modulus, $A$ is the cross section area of tendon, $\lambda$ is a non-dimensional parameter and indicates the total friction force acting on the tendon under unit tendon-tension[2]. We can regard $\delta(L)$ as a kind of backlash for the tendon-sheath driving system. For any tendon-sheath driving system, $L, E, A$ can be considered as constant. $\lambda$ could also be considered as a constant if the curve of the tendon-sheath doesn't change much. From equation (5), the backlash length is only a function of the cable tension $T_{0}$. To improve the performance of the system, a pretension device is introduced so that the tendon is always in tension. The modified mechanism [14] could also minimize the problem of wire elongation after repeated use.

The pretension is set in such a way that the tension change during robot movement is trivial compared to the pretension, so that backlash length $\delta(L)$ can be considered as a constant.

To further reduce the movement delay of the slave the software will record the movement of each slave joint, whenever a direction change is detected, additional actuator displacement is used to compensate for the backlash. Experiments have been designed and performed to obtain the appropriate backlash compensation displacement for each joint. Using this method the delay of the slave can be reduced by up to 0.5 second.

\section{V.CONCLUSIONS}

A master slave robotic system that could enhance gastrointestinal endoscopic procedures has been designed and built. The developed slave robotic system consists of a long and flexible body that allows it to follow the endoscope through the human natural orifice. This characteristic has the potential to allow treatment such as suturing to be performed to the patient without the need of any incision. User has been able to apply the master-slave system to perform tasks such as grabbing and cutting as well as pick and place. Animal trials are planned using the developed system. Future work includes incorporating force sensors and haptic devices into the robotic system. The aim is to enable the endoscopist to 'feel' as if the slave manipulators are his own hands. 


\section{REFERENCES}

[1] L. Phee, W.S. Ng, I.M. Chen, F. Seow-Cheon and B.L. Davies; Locomotion and Steering Aspects in Automation of Colonoscopy; IEEE Engineering in Medicine and Biology Magazine, U.S.A., Nov 1997, Vol. 16(6), pp. 85-96

[2] Intuitive Surgical Inc. Available: http://www.intuitivesurgical.com/

[3] M.C. Carrozza, P. Dario and L. Phee; Micromechatronics in Surgery; Transactions of the Institute of Measurements and Control, England, Sep 2003, Vol. 25(4), pp. 309-327

[4] J.P. Friconneau, P. Garrec, F. Gosselin and A. Riwan; Status and trends in force feedback master arm at CEA, IARP workshop, Rome, 2002

[5] M. Tavakoli, R.V. Patel and M. Moallem; Design issues in a Haptics-Based Master-Slave System for Minimally Invasive Surgery; IEEE Conference on Robotics \& Automation, New Orleams, L.A., April 2004, pp. 371-376

[6] S.C. Low and L. Phee; A Review of Master-Slave Robotic Systems for Surgery; IEEE Conference on Robotics, Automation and Mechatronics, Singapore Dec 2004, pp. $37-42$

[7] V. Hayward, P. Gregorio, O. Astley, S. Greenish and M. Doyon; Freedom-7: A High Fidelity Seven Axis Haptic Device With Application To Surgical Training; Experimental Robotics; Lecture Notes in Control and Information Science 232, Springer-Verlag, 1998, pp. 445456

[8] M. Mitsuishi, J. Arata, K. Tanaka, M. Miyamoto, T. Yoshidome, S. Iwata, M. Hashizume and S. Warisawa; Development of a remote minimally-invasive surgical system with operational environment transmission capability; IEEE International Conference on Robotics and Automation, 14-19 Sept 2003, Volume 2, pp. 2663-2670

[9] R. Tadakuma, Y. Asahara, H. Kajikoto, N. kawakami and S. Tachi; Development of Anthropomorphic Multi-D.O.F. Master-Slave Arm for Mutual Telexistence; IEEE Transactions on Visualization and Computer Graphics, Nov/Dec 2005, Volume 11, pp. 626-636

[10] Sensory-Feedback Exoskeletal Arm Controller. Available: http://www.nasatech.com/Briefs/Apr04/MSC22563.html

[11] N. Stanton, A. Hedge, K. Brookhuis, E. Salas and H. Hendrick; Handbook of Human Factors and Ergonomics Methods; CRC Press LLC, 2005

[12] Estimation Method of Center of Rotation of Functional Shoulder Joint. Available: http://www.dh.aist.go.jp/research/centered/shoulder/index.p hp.en

[13] J. J. Craig; Introduction to Robotics: Mechanics and Control; $3^{\text {rd }}$ Edition, Prentice Hall, 2005

[14] Kaneko. M, Yamashita. T, Tanie. K; Basic considerations on transmission characteristics for tendon drive robots; Advanced Robotics 1991, 'Robots in Unstructured Environments', 91 ICAR, Fifth International Conference; 19-22 June 1991 pp 827 - 832 vol.1 